\title{
Experimental Studies of Hadron Structure via Generalized Parton Distributions
}

\author{
Silvia Niccolai ${ }^{* \dagger}$ \\ IPN Orsay \\ E-mail: niccolai@ipno.in2p3.fr
}

Generalized Parton Distributions (GPDs) provide a unified description of hadronic structure in terms of quark and gluonic degrees of freedom. Deeply virtual Compton scattering (DVCS) is the key reaction to gain information on GPDs. In this article recent results of DVCS from Jefferson Lab, as well as those planned for the near future, are presented and discussed.

The 26th International Nuclear Physics Conference

11-16 September, 2016

Adelaide, Australia

${ }^{*}$ Speaker.
${ }^{\dagger}$ CLAS Collaboration 


\section{Introduction}

Our understanding of nucleon structure has deepened recently thanks to the ongoing research on Generalized Parton Distributions (GPDs). These structure functions describe the correlations between the longitudinal momentum and transverse spatial position of the partons inside the nucleon, and, among other features, they give access to the contribution of the orbital momentum of the quarks and gluons to the nucleon [1-9]. The nucleon GPDs are accessible via measurements of

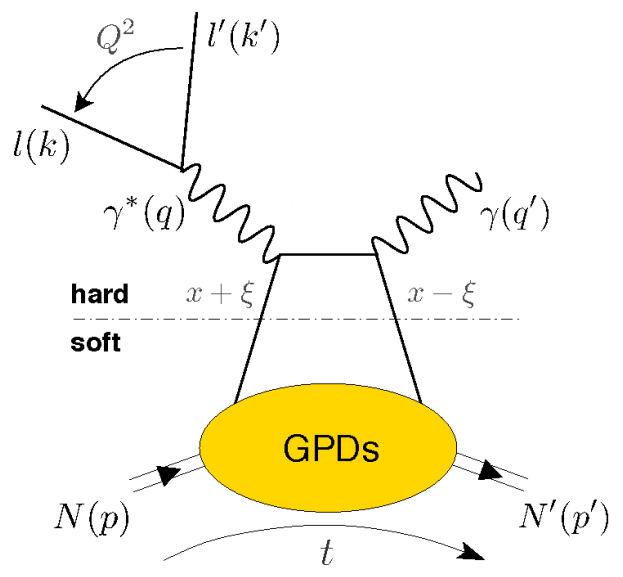

Figure 1: The handbag diagram for the DVCS process on the nucleon $\ell N \rightarrow \ell^{\prime} N^{\prime} \gamma$. The four-momenta of the incoming and outgoing leptons are labeled as, respectively, $k$ and $k^{\prime}$, while those of the virtual and real photons are indicated by, respectively, $q$ and $q^{\prime} . x$ is the average longitudinal momentum fraction of the struck parton, and $\xi$ is the difference of longitudinal momentum fractions between the initial and the final parton. $t=\left(p-p^{\prime}\right)^{2}$ is the squared four-momentum transfer between the initial and final nucleon. The four-momenta of the virtual and real photons are indicated by, respectively, $q$ and $q^{\prime}$.

hard exclusive leptoproduction of a real photon, $\ell N \rightarrow \ell^{\prime} N^{\prime} \gamma$ (where the $\gamma^{*} N \rightarrow N^{\prime} \gamma$ subprocess is called deeply virtual Compton scattering, DVCS), or of a meson (deeply virtual meson production, DVMP). This article will be devoted to the DVCS reaction. Figure 1 illustrates the leading process for DVCS, also called "handbag diagram". The virtual photon interacts with a quark of the nucleon having a longitudinal momentum fraction $x+\xi$. The quark is then reabsorbed in the nucleon with a momentum fraction $x-\xi$, and a real photon is emitted (Fig. 1). Conditions are required for the applicability of the GPD formalism and the validity of the handbag approximation. More precisely, the virtuality $Q^{2}\left(Q^{2}=-q^{2}=-\left(k-k^{\prime}\right)^{2}\right)$ of the exchanged photon between the initial lepton and the nucleon must be sufficiently large for the reaction to happen at the parton level, and much larger than the squared four-momentum transfer to the target $t,\left(|t| / Q^{2}<<1\right)$. In these conditions, the cross section of the DVCS process can be expressed as a convolution of a hard partonic subprocess, that is calculable in perturbative QED, and a soft nucleon-structure part, parametrized by the GPDs. At leading order in $\alpha_{S}$ and at leading twist, the GPDs depend upon three variables, $x, \xi$, and $t$, where the skewness $\xi$ is related to the Bjorken variable $x_{B}\left(x_{B}=\frac{Q^{2}}{2 M v}\right)$, as $\xi \simeq \frac{x_{B}}{2-x_{B}}$. The reaction is described by four GPDs, $H^{q}, \tilde{H}^{q}, E^{q}, \tilde{E}^{q}$, that account for the possible combinations of relative orientations of nucleon spin and parton helicity between the initial and final state. The GPDs $H$ and $E$ do not depend on the parton helicity and are therefore called unpolarized GPDs, while $\tilde{H}$ 
and $\tilde{E}$ depend on the parton helicity and are called polarized GPDs. The GPDs $H$ and $\tilde{H}$ conserve the spin of the nucleon, whereas $E$ and $\tilde{E}$ correspond to a nucleon-spin flip. At $\xi=0$, the Fourier transform of the $t$ dependence of a GPD provides the spatial distribution in the transverse plane for partons having a longitudinal momentum fraction $x$ [6]. Model-independent sum rules link the first moment in $x$ of the GPDs to the elastic form factors (FFs) [2]. In the forward limit $(t \rightarrow 0)$, the second moment in $x$ of the sum of the GPDs $H$ and $E$ is linked to the total angular momentum carried by the quarks, $J_{q}$, via Ji's sum rule [2].

The DVCS final state is indistinguishable from that of the well-known Bethe-Heitler (BH) process in which the final-state photon is radiated by the incoming or scattered lepton and not by the nucleon itself. The two processes interfere, and the cross section of the hard exclusive leptoproduction of a real photon is proportional to the sum of the squared DVCS and BH amplitudes, plus an interference term $(I)$. Given that the nucleon form factors are well known at small $t$, the BH amplitude $T_{B H}$ is precisely calculable theoretically. Eight GPD-related quantities can be extracted from DVCS: the real and imaginary parts of four Compton Form Factors (CFFs), defined as:

$$
\begin{aligned}
& \Re \mathrm{e} \mathscr{F}(\xi, t)=F_{R e}(\xi, t)=\sum_{q} e_{q}^{2} \mathscr{P} \int_{-1}^{1} d x\left[\frac{1}{x-\xi} \mp \frac{1}{x+\xi}\right] F^{q}(x, \xi, t), \\
& \mathfrak{I m} \mathscr{F}(\xi, t)=-\pi F_{I m}(\xi, t)=-\pi \sum_{q} e_{q}^{2}\left[F^{q}(\xi, \xi, t) \mp F^{q}(-\xi, \xi, t)\right],
\end{aligned}
$$

where $\mathscr{P}$ is Cauchy's principal value integral, $F$ represents a generic nucleon GPD and the top and bottom signs apply, respectively, to the unpolarized GPDs $(H, E)$ and to the polarized GPDs $(\widetilde{H}, \widetilde{E})$. DVCS observables can be expressed as linear and bilinear combinations of CFFs, electromagnetic form factors, and terms depending on the kinematics. The study, at fixed $Q^{2}, x_{B},-t$, of the $\phi$ dependence of DVCS cross sections, cross-section differences, and asymmetries can help single out the contributions from the various CFFs. Four-fold $\left(Q^{2}, x_{B},-t, \phi\right)$ differential cross sections, unpolarized and beam-polarized, as well as single and double spin asymmetries with polarized target, for the exclusive electroproduction of photons were recently measured at Jefferson Lab, by the Hall A and the CLAS Collaborations.

\section{Hall A results}

E00-110, the first experiment specifically dedicated to DVCS, ran in Hall A at Jefferson Lab during the year 2004, with the primary goal of performing a $Q^{2}$-scaling test of the DVCS cross section [15]. Polarized electrons, accelerated to $5.7572 \mathrm{GeV}$ by the CEBAF accelerator, impinged on a 15-cm-long liquid-hydrogen target. The exclusivity of the $e p \gamma$ final state was ensured by detecting the scattered electrons in the High Resolution Spectrometer, the DVCS/BH photons in a custombuilt $\mathrm{PbF}_{2}$ electromagnetic calorimeter, and reconstructing the recoil protons via the $e p \rightarrow e \gamma X$ missing mass. ep $\gamma$ events were collected for five different $\left(Q^{2}, x_{B}\right)$ kinematics, which were further differentiated in $5-t$ bins and $24 \phi$ bins, and four-fold unpolarized and beam-polarized cross sections were extracted. The left plot of Fig. 2 shows the obtained results for the $-t=0.23 \mathrm{GeV}^{2}$ bin of Kin2 $\left(Q^{2}=1.9 \mathrm{GeV}^{2}, x_{B}=0.36\right)$. The different contributions to the cross section (DVCS, $\mathrm{BH}$, Interference), were separated, up to twist three for the interference term, using the BMK formalism [16]. This method also allowed the extraction of five observables linked to combinations 
of CFFs. Each of these observables isolates the real or the imaginary part of a distinct combination of linear $\left(B H \cdot D V C S^{\dagger}\right)$ and bilinear $\left(D V C S \cdot D V C S^{\dagger}\right)$ terms. The constant $Q^{2}$ dependence of the five observables (Fig. 2, right) confirms that the factorization and leading-twist approximations for DVCS are valid already at these relatively low $Q^{2}\left(\sim 1-2(\mathrm{GeV} / \mathrm{c})^{2}\right)$, as first observed in the previous analysis of these same data [12]. In the left part Fig. 2, the results are compared to double-
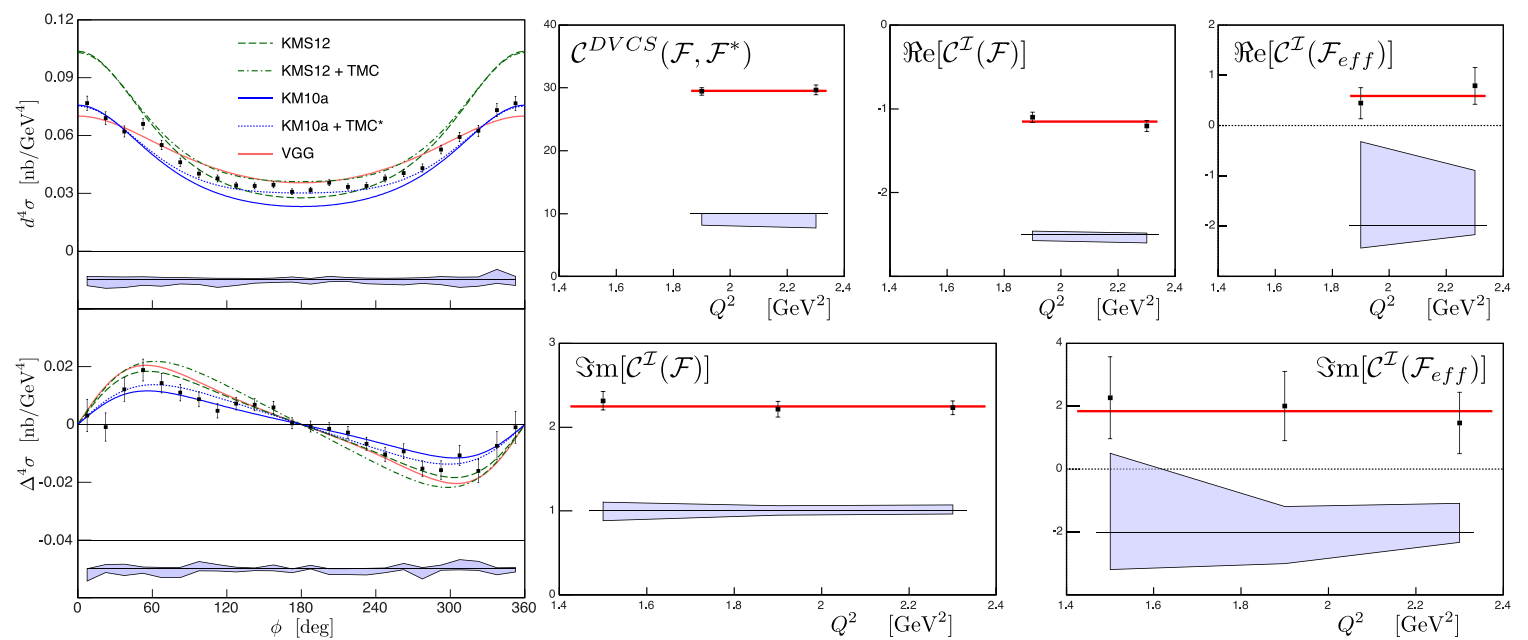

Figure 2: Left: unpolarized (top) and helicity-dependent (bottom) cross sections for the Hall-A Kin2 bin, $t=0.23 \mathrm{GeV}^{2}$. The light blue area represents the systematic uncertainties. The predictions from the KMS12 and VGG models are shown as the dashed green and solid red curves, respectively. The KM10a fit is represented as the solid blue line. Target-mass and finite- $t$ corrections are included in the KMS12 model and shown as the dotted-dash curve. The correction is then applied to the KM10a model shown as the dotted blue line. Right: combinations of effective CFFs extracted from the Hall-A data, integrated over $t$ and plotted as a function of $Q^{2}$. The top three plots show the effective CFFs resulting from the unpolarized cross section fit (Kin2 and Kin3), whereas the bottom plots show the effective CFFs resulting from the helicity-dependent cross section fit (Kin1-3). The shaded areas represent systematic errors. Figures taken from Ref. [15].

distribution GPD models (VGG [7, 10,14,17] and KMS12 [18]) and fits to previous data. Unlike VGG, the KMS12 model was tuned using vector meson data at low to very-low $x_{B}$, and may be not optimal for the valence-quark region. Both models overestimate the helicity-dependent cross sections in this kinematics, while VGG provides a better fit for the unpolarized data. The data are also compared to the KM10a model [13], which reproduces very well the helicity-dependent data but underestimates significantly the DVCS unpolarized cross section around $\phi=180^{\circ}$. Adding an empirical estimate of the target-mass and finite- $t$ corrections to the KM10a model improves the agreement with the data significantly. This may hint at the necessity to include such effects in the analysis of moderate- $Q^{2}$ data, which is relevant for current and future Jefferson Lab experiments.

A new DVCS experiment, with similar setup of E00-110 but using the upgraded CEBAF accelerator, ran recently in Hall A at Jefferson Lab [11]. It aims to measure the $\vec{e} p \rightarrow e p \gamma$ cross sections at fixed $x_{B}$ over as wide a range in $Q^{2}$ as possible for $k \leq 11 \mathrm{GeV}$, by using three different electron-energy settings. This will determine at what level the "handbag" amplitude dominates, or not, over the higher-twist amplitudes. The expected accuracy for the absolute cross sections and beam-helicity cross-section differences will allow to extract five kinematically independent observables, for each $Q^{2}, x_{B}, t$ point, which are angular harmonic superpositions of CFFs as functions of 
$\phi$, similarly to what was done for E00-110 (see Fig. 2, right). The $t$-dependence of each angular harmonic term, linked to the Fourier conjugate of the transverse spatial distribution of the quarks in the nucleon, will also be measured, as a function of the quarks' momentum fraction $x$. A similar experiment, which will complement the kinematic coverage of the Hall A, is also planned in Hall C at Jefferson Lab [19].

\section{CLAS results on unpolarized target}

The "e1-dvcs" experiment took place at JLab during three months in 2005, using the CEBAF 5.75-GeV polarized electron beam (79.4\% polarization), a $2.5-\mathrm{cm}$-long liquid hydrogen target, and the Hall B large-acceptance CLAS spectrometer [20], operating at a luminosity of $2 \cdot 10^{34} \mathrm{~cm}^{2} \mathrm{~s}^{-1}$. The polar-angle acceptance of CLAS for photons $\left(15-45^{\circ}\right)$ was extended down to $5^{\circ}$ with the addition of a specially designed electromagnetic calorimeter ("inner calorimeter", IC [21]), with full azimuthal coverage. ep $\gamma$ events were detected in CLAS and in the IC, and tight cuts on missing masses and angles ensured the exclusivity of the final state. The data were divided into $21\left(Q^{2}, x_{B}\right)$ bins, $6-t$ bins, and 24 bins in $\phi$. Figure 3 shows, for a selected $\left(Q^{2}, x_{B}\right)$ bin and three $-t$ bins are shown, the $\phi$-dependence of the $e p \rightarrow e^{\prime} p^{\prime} \gamma$ unpolarized cross section (top) and beam-polarized cross-section difference (bottom). The unpolarized cross sections peak towards $\phi=0^{\circ}$ due to the

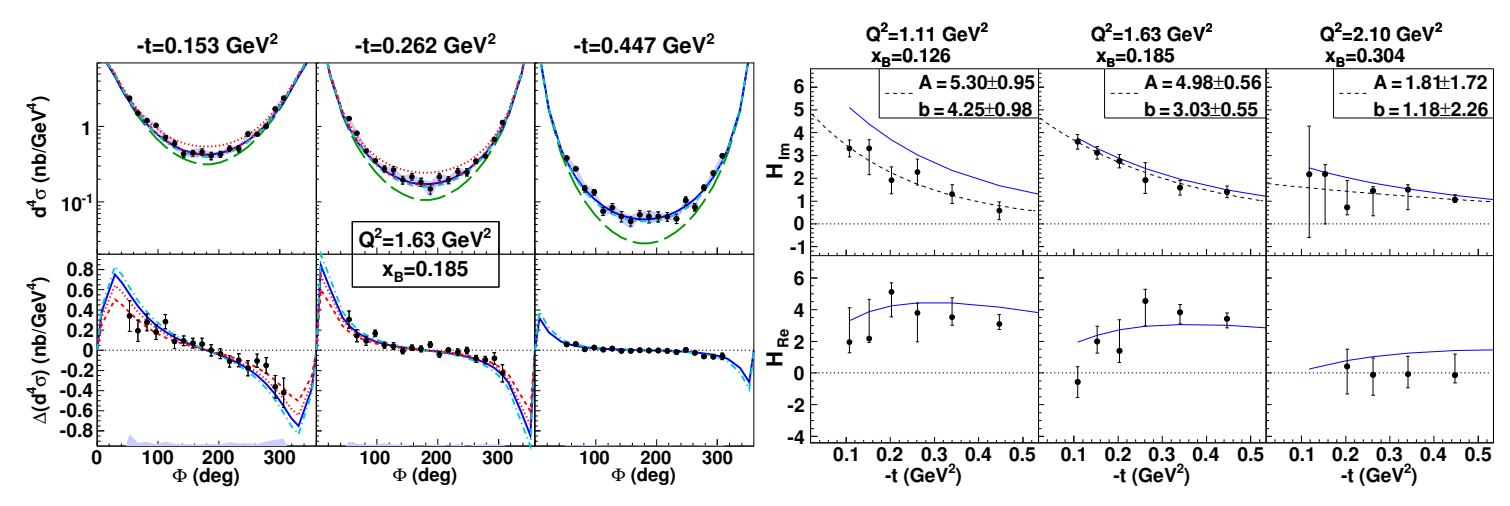

Figure 3: Left: unpolarized cross section (top row) and beam-polarized cross-section difference for the $e p \rightarrow e^{\prime} p^{\prime} \gamma$ reaction, as a function of $\phi$, for a particular $\left(Q^{2}, x_{B}\right)$ bin and for 3 selected values of $-t$. The green long-dashed curves show the $\mathrm{BH}$ contribution only. The other curves correspond to the predictions of four GPD models: VGG [7, 10, 14, 17] (blue solid curves), KMS [18] (cyan dash-dotted curves), and two versions of the KM model [13,24], KM10 (red dotted curves) and KM10a (red short-dashed curves). The blue bands show the systematic uncertainties. Right: results of the CFF fit of the CLAS unpolarized and beam-polarized cross sections for $H_{I m}$ (upper panel) and $H_{R e}$ (lower panel), for three $\left(Q^{2}, x_{B}\right)$ bins, as a function of $t$. The blue solid curves are the predictions of the VGG model. The black dashed curves show the fit of the results by the function $A e^{b t}$. Figures taken from Ref. [22].

BH process (green long-dashed curves in the left side of Fig. 3). The difference between the BH curves and the data can thus be attributed to the DVCS process. The left plot of Fig. 3 shows also the calculations of four GPD models, described in the caption. Three of these models, VGG, $\mathrm{KMS}$, and the KM10a version of the KM model, describe the data well without additional inputs, while the KM10 model, which contains a sizeable contribution from $\widetilde{H}$, fails to reproduce the data 
near $\phi=180^{\circ}$. Therefore, the e1-dvcs results reinforce the expectation of the $H$-dominance in the unpolarized ep $\gamma$ cross section.

These data were also used to extract the CFFs, using the procedure described in $[25,26]$, based on a local-fitting method at each given experimental $\left(Q^{2}, x_{B},-t\right)$ kinematic point. The conditions adopted to fit the CLAS cross sections are described in Ref. [22]. The right plot of Fig. 3 shows, for a selection of three out of the $21\left(Q^{2}, x_{B}\right)$ bins, the $t$ distribution of $H_{I m}$ and $H_{R e}$, along with the predictions of the VGG model, which overestimates the fitted $H_{I m}$ at the smallest values of $x_{B}$. The $t$-dependence of $H_{I m}$ was fitted with the function $A e^{b t}$, with $A$ and $b$ left as free parameters. Reminding that $b$ is related to the transverse size of the nucleon, the fact that $A$ and $b$ increase with decreasing $x_{B}$ suggests that the nucleon size increases at lower parton-momentum values, thus revealing from experiment a first tomographic image of the nucleon. This image was confirmed and refined by a more accurate recent analysis [23].

\subsection{CLAS results on polarized target}

The data, part of the "eg1-dvcs" experiment which ran in Hall B at Jefferson Lab during the year 2009 , were produced by a $5.9-\mathrm{GeV}$ polarized electron beam impinging onto a solid ammonia target $\left(\mathrm{NH}_{3}\right)$, polarized along the beam direction [29], and collected by the CLAS detector. Exclusive ep $\gamma$ events were extracted, applying cuts on missing masses and angles formed with the quadrivectors of the detected electron, proton, and photon. These cuts served the dual purpose of suppressing the background of events scattered from the nuclear target $\left(N_{14}\right)$ and the ep $\pi^{0}$ events, with only one decay photon reconstructed, mimicking the $e p \gamma$ final state. The phase space covered by the selected events was divided into 21 bins in $\left(Q^{2}, x_{B},-t\right)$. For each $\left(Q^{2}, x_{B},-t\right)$ bin, beam-, target-, and double spin asymmetries were extracted as a function of $\phi$, in ten equidistant bins. The two single-spin asymmetries were fitted with the $\frac{\alpha \sin \phi}{1+\beta \cos \phi}$ function, according to the leading-twist harmonic expansion. The left plot of Fig. 4 shows the parameter $\alpha$ for the TSA as a function of $-t$, for the five measured $Q^{2}-x_{B}$ bins, compared to four GPD models, described in the caption. The amplitude of the target-spin asymmetry seems rather constant as a function of all kinematic variables, $-t$ included, apart from the expected systematic drop towards $t \sim t_{\min }$. This trend is quite different from what is observed for the beam-spin asymmetry, which is found to be consistent with the the one previously measured at CLAS [21], which display a much stronger drop, by about a factor of 3 on average, for all $Q^{2}-x_{B}$ kinematics but more markedly at low $x_{B}$. The DVCS/BH beam-spin and target-spin asymmetries are mostly sensitive to the GPDs $H$ and to a combination of $\tilde{H}$ and $H$, respectively. Therefore, considering that the $t$-slope of the GPDs is linked via a Fourierlike transform to the transverse position of the struck parton [5], this result suggests that the axial charge (linked to $\mathfrak{I m} \tilde{\mathscr{H}}$ ) is more concentrated in the center of the nucleon than the electric charge (linked to $\mathfrak{I m} \mathscr{H}$ ), confirming what was first observed in [25]. This is in agreement with the behavior as a function of $Q^{2}$ of the axial form factor, which is the first moment in $x$ of $\widetilde{H}$, and which was measured in $\pi^{+}$electroproduction experiments on the proton as well as in neutrino-nucleon scattering [32]. These results adds to this the extra information on the longitudinal momentum of the partons. The flat $-t$ slope of the target-spin asymmetry is clearly not predicted by the VGG or KMS models, which instead display a similar drop with $t$ for the TSA as what was computed for the BSA. These models approximately reproduce the low- $t$ magnitude of the asymmetry in some kinematics, with a slightly better fit of the data for VGG. KMS predicts an increase of the TSA with 
$x_{B}$ that is not observed in the experiment. The GGL model also predicts a drop in $-t$ not confirmed by the data, and moreover it overestimates the magnitude of the asymmetry by at least a factor of 2. The best fit to the data is provided, in the bins where it applies, by the KMM12 model.
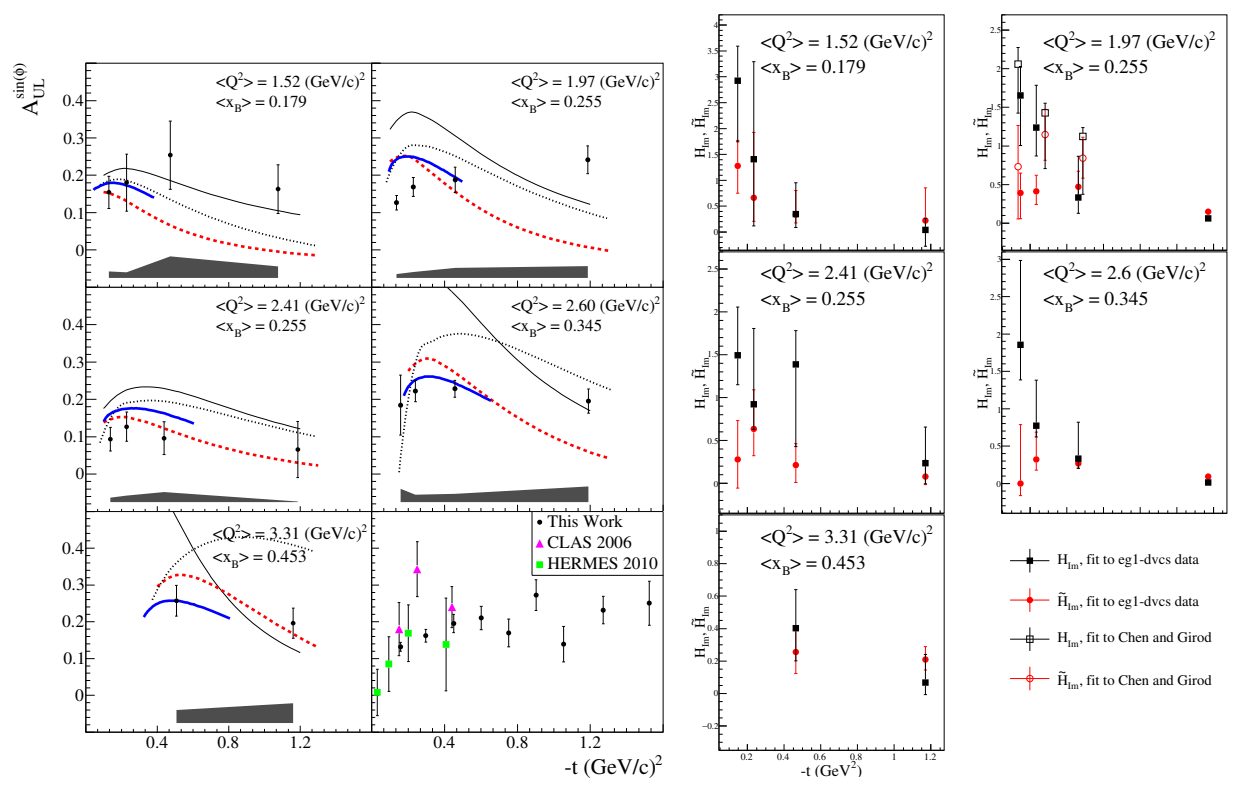

Figure 4: Left: first five plots: $-t$ dependence of the $\sin \phi$ amplitude of the TSA ( $A_{\mathrm{UL}}$ ) for each $Q^{2}-x_{B}$ bin. The shaded bands represent the systematic uncertainties. The curves show the predictions of four GPD models: i) VGG [10,17] (red-dashed), ii) GK [18] (black-dotted), KMM12 [30] (blue-thick solid), GGL [27,33] (black-solid). Bottom right plot: comparison of the $\sin \phi$ amplitude of $A_{\mathrm{UL}}$ as a function of $-t$ for the results of this work (black dots) integrated over all $Q^{2}$ and $x_{B}$ values $\left(\left\langle Q^{2}\right\rangle=2.4(\mathrm{GeV} / \mathrm{c})^{2}\right.$, $\left\langle x_{B}\right\rangle=0.31$ ), the HERMES results [34] (green squares) at $\left\langle Q^{2}\right\rangle=2.459(\mathrm{GeV} / \mathrm{c})^{2},\left\langle x_{B}\right\rangle=0.096$, and the previously published CLAS results [35] (pink triangles), at $\left\langle Q^{2}\right\rangle=1.82(\mathrm{GeV} / \mathrm{c})^{2},\left\langle x_{B}\right\rangle=0.28$. The figure is from Ref. [36]. Right: $t$ dependence for each $Q^{2}-x_{B}$ bin of $H_{I m}$ (black squares) and $\tilde{H}_{I m}$ (red circles). The full points are obtained by fitting the eg1-DVCS data (TSA, BSA and DSA) [31]. The empty points were obtained by fitting the BSA results from [21] integrated over all values of $Q^{2}$ at $x_{B} \sim 0.25$, and the TSAs from [35]. Figure taken from Ref. [31].

The three sets of asymmetries (BSA, TSA and DSA) for all kinematic bins were processed using the fitting procedure described in $[25,26]$ to extract the CFFs. The right plot of Fig. 4 shows $H_{I m}$ (black full squares) and $\tilde{H}_{I m}$ (red full circles), which are obtained from the fit of the CLAS eg1-dvcs data, as a function of $-t$ for each of the $5 Q^{2}-x_{B}$ bins. These are the two CFFs that appear to be better constrained by these results. Given that the size of the error bars reflects the sensitivity of the combination of observables to each CFF, it is evident that, as expected, these asymmetries are mostly sensitive to $\mathfrak{I m} \tilde{\mathscr{H}}$. The results for $H_{I m}$ and $\tilde{H}_{I m}$ confirm what had been previously observed in a qualitative way by direct comparison of the $t$ dependence of TSAs and BSAs: the $t$-slope of $\mathfrak{I m} \mathscr{H}$ is much steeper than that of $\mathfrak{I} \mathrm{m} \tilde{\mathscr{H}}$, hinting at the fact that the axial charge (linked to Im $\tilde{\mathscr{H}}$ ) might be more "concentrated" in the center of the nucleon than the electric charge (linked to $\mathfrak{I m} \mathscr{H}$ ). This effect seems stronger at the lowest values of $x_{B}$, while both CFFs tend to flatten out as $x_{B}$ increases. It is also interesting to compare the results obtained for the two equal- $x_{B}$ bins 
$\left(Q^{2}=1.97(\mathrm{GeV} / c)^{2}\right.$ and $\left.Q^{2}=2.41(\mathrm{GeV} / c)^{2}\right)$ : within the limits imposed by the size of the error bars and by the $Q^{2}$ lever arm (only $\left.0.44(\mathrm{GeV} / c)^{2}\right)$, both sets of CFFs are compatible, at the 1- $\sigma$ level, which supports the validity of the scaling hypothesis. In the right plot of Fig. 4 the values of $H_{I m}$ and $\tilde{H}_{I m}$ that were obtained [37] using the same fitting code with the results from e1-dvcs [21] for the beam-spin asymmetry and from a previous polarized-target CLAS experiment [35] for the target-spin asymmetry, are also shown. Aside from the much larger kinematic coverage for the polarized-target observables made accessible by the new data, in the kinematics where the previous extraction had been attempted, they improve the precision of $\mathfrak{I} \mathrm{m} \tilde{\mathscr{H}}$.

The eg1-dvcs results brought important new information on the $x_{B}$ and $t$ dependence of CFFs, especially $\mathfrak{I} \mathrm{m} \tilde{\mathscr{H}}$, on quite a wide kinematical coverage, but their low statistics allowed to perform only a rather coarse four-dimensional binning. This shortcoming will be compensated by the planned CLAS12 experiment for DVCS at $11 \mathrm{GeV}$ on a longitudinally polarized ammonia target [28]. The precision and coverage that will be obtained for the target-spin asymmetry, which will permit a precise tuning of GPD parametrizations, in particular for $\tilde{H}$. The double-spin asymmetry $A_{L L}$ will be a by-product of this experiment, bringing sensitivity to the real part of CFFs.

\section{DVCS on the neutron}

Measuring Deeply Virtual Compton Scattering on a neutron target is one of the necessary steps to complete our understanding of the structure of the nucleon in terms of GPDs. Extracting neutron GPDs is complementary to extracting proton GPDs, as it allows to carry out a quark-flavor separation. The importance of neutron targets in the DVCS phenomenology was clearly established in the pioneering Hall A experiment, which has provided, at today, the only existing data set for neutron DVCS [39]. Albeit these results were limited by their small kinematical coverage, low statistical precision and high systematic uncertainties mostly coming from the subtraction of hydrogen data from deuterium ones, they allowed, for the first time, a model-dependent extraction of $J_{u}$ and $J_{d}$, combined to HERMES results for proton-DVCS on transversely polarized target. The experiments planned at JLab at $12 \mathrm{GeV}[38,40]$ will have the ultimate goal to achieve a model-independent measurement of quark-flavor separated CFFs, achieved by combining the obtained neutron CFFs with those that will be extracted from the proton-DVCS measurements. The flavor separation of the CFFs will represent a major step forward towards the unraveling of the contribution of the quarks' angular momentum to the total nucleon spin via Ji's sum rule.

\section{References}

[1] D. Mueller, D. Robaschik, B. Geyer, F. M. Dittes, and J. Hoeji, Fortsch. Phys. 42, 101 (1994).

[2] X.-D. Ji, Phys. Rev. Lett. 78, 610 (1997); Phys. Rev. D55, 7114 (1997).

[3] A. V. Radyushkin, Phys. Lett. B380, 417 (1996); Phys. Rev. D56, 5524 (1997).

[4] J. C. Collins, L. Frankfurt, and M. Strikman, Phys. Rev. D56, 2982 (1997).

[5] M. Burkardt, Phys. Rev. D62, 071503 (2000), [Erratum: Phys. Rev.D66,119903(2002)]; Int. J. Mod. Phys. A18, 173 (2003),

[6] M. Burkardt, Phys. Lett. B595, 245 (2004). 
[7] K. Goeke, M. V. Polyakov, and M. Vanderhaeghen, Prog. Part. Nucl. Phys. 47, 401 (2001).

[8] M. Diehl, Phys. Rept. 388, 41 (2003).

[9] A. V. Belitsky and A. V. Radyushkin, Phys. Rept. 418, 1 (2005).

[10] M. Vanderhaeghen, P. A. M. Guichon, and M. Guidal, Phys. Rev. Lett. 80, 5064 (1998).

[11] Hall A, C. Hyde et al., Jefferson Lab experiment E12-06-114.

[12] Jefferson Lab Hall A, C. M. Camacho et al., Phys. Rev. Lett. 97, 262002 (2006).

[13] K. Kumericki and D. Mueller, Nucl. Phys. B841, 1 (2010).

[14] M. Guidal, M. V. Polyakov, A. V. Radyushkin, and M. Vanderhaeghen, Phys. Rev. D72, 054013 (2005).

[15] Jefferson Lab Hall A, M. Defurne et al., Phys. Rev. C92, 055202 (2015).

[16] A. V. Belitsky and D. Mueller, Phys. Rev. D82, 074010 (2010).

[17] M. Vanderhaeghen, P. A. M. Guichon, and M. Guidal, Phys. Rev. D60, 094017 (1999).

[18] P. Kroll, H. Moutarde, and F. Sabatie, Eur. Phys. J. C73, 2278 (2013).

[19] Hall C, C. Munoz et al., Jefferson Lab experiment E12-13-010.

[20] CLAS, B. A. Mecking et al., Nucl. Instrum. Meth. A503, 513 (2003).

[21] CLAS, F. X. Girod et al., Phys. Rev. Lett. 100, 162002 (2008).

[22] CLAS, H. S. Jo et al., Phys. Rev. Lett. 115, 212003 (2015).

[23] R. Dupre, M. Guidal, and M. Vanderhaeghen, Phys. Rev. D95, 011501 (2017).

[24] K. Kumericki et al., (2011), 1105.0899.

[25] M. Guidal, Eur. Phys. J. A37, 319 (2008), [Erratum: Eur. Phys. J.A40, 119(2009)].

[26] M. Guidal, H. Moutarde, and M. Vanderhaeghen, Rept. Prog. Phys. 76, 066202 (2013).

[27] G. R. Goldstein, J. O. Hernandez, and S. Liuti, Phys. Rev. D84, 034007 (2011).

[28] CLAS12, F. Sabatie et al., Jefferson Lab experiment E12-06-119.

[29] C. D. Keith et al., Nucl. Instrum. Meth. A501, 327 (2003).

[30] K. Kumercki, D. Muller, and M. Murray, Phys. Part. Nucl. 45, 723 (2014).

[31] CLAS, S. Pisano et al., Phys. Rev. D91, 052014 (2015).

[32] A. Bodek, S. Avvakumov, R. Bradford, and H. S. Budd, Eur. Phys. J. C53, 349 (2008).

[33] J. O. Gonzalez-Hernandez, S. Liuti, G. R. Goldstein, and K. Kathuria, Phys. Rev. C88, 065206 (2013).

[34] HERMES, A. Airapetian et al., JHEP 06, 019 (2010).

[35] CLAS, S. Chen et al., Phys. Rev. Lett. 97, 072002 (2006).

[36] CLAS, E. Seder et al., Phys. Rev. Lett. 114, 032001 (2015), [Addendum: Phys. Rev. Lett.114, no.8, 089901 (2015)].

[37] M. Guidal, Phys. Lett. B689, 156 (2010).

[38] CLAS12, L. Elouadrhiri et al., Jefferson Lab experiment C12-12-010.

[39] Jefferson Lab Hall A, M. Mazouz et al., Phys. Rev. Lett. 99, 242501 (2007).

[40] CLAS12, S. Niccolai et al., Jefferson Lab experiments E12-11-003 and E12-06-109a. 